\title{
Stability of Tautological Vector Bundles on Hilbert Squares of Surfaces
}

\author{
ULRICH SCHLICKEWEI
}

\begin{abstract}
We prove stability of rank two tautological bundles on the Hilbert square of a surface (under a mild positivity condition) and compute their Chern classes.
\end{abstract}

Let $S$ be a smooth, complex projective surface, let $\operatorname{Hilb}^{2}(S)$ be the Hilbert scheme parametrizing subschemes of $S$ of length 2. It is known by a classical theorem of Fogarty $\left[\mathrm{Fo}_{0}\right]$ that $\operatorname{Hilb}^{2}(S)$ is a smooth, projective variety of dimension 4 . Let $Z \subset S \times \operatorname{Hilb}^{2}(S)$ be the universal subscheme, denote by $p: Z \rightarrow S$ and by $q: Z \rightarrow \operatorname{Hilb}^{2}(S)$ the projections. Given a line bundle $\mathcal{L}$ on $S$, the sheaf $\mathcal{L}^{[2]}:=q_{*} p^{*} \mathcal{L}$ is a rank two vector bundle on $\operatorname{Hilb}^{2}(S)$, called the tautological vector bundle associated with $\mathcal{L}$.

In this note we prove the following

Theorem 1. Assume that $h^{0}(S, \mathcal{L}) \geq 2$. Then for $N \gg 0$, the vector bundle $\mathcal{L}^{[2]}$ is $\mu_{H_{N}}$-stable on $\operatorname{Hilb}^{2}(S)$.

Here, $H_{N}$ is a polarization of the form $\operatorname{Sym}^{2}(N H)-E$ where $H$ is an ample divisor on $S$ and $E \subset \operatorname{Hilb}^{2}(S)$ denotes the exceptional divisor of the Hilbert-Chow morphism. The proof of the theorem relies upon the fundamental short exact sequence for tautological vector bundles on the blowup of $S \times S$ and upon the corresponding result for curves which was proved by Mistretta [Mi].

Indirizzo dell'A.: Technische Universität München, Fakultät für Informatik, Boltzmannstr. 3, 85748 Garching, Germany

E-mail: ulrich.schlickewei@in.tum.de 
Originally, our interest in this result came from the desire to produce vector bundles on Hilbert schemes of K3 surfaces with interesting metrics and with interesting Chern classes. For this reason we give a formula for the Chern classes of $\mathcal{L}^{[2]}$ in terms of the symmetric product of $\mathrm{c}_{1}(\mathcal{L})$, of $[E]$ and of the characteristic classes of $\operatorname{Hilb}^{2}(S)$.

Moduli spaces of stable sheaves on K3 surfaces have been studied extensively in the literature (cf. e.g. [Mu], [OG], [HL], [Ma]). These spaces are particularly interesting because they are among the few examples of compact Hyperkähler manifolds (cf. Huybrechts' chapter in [GHJ]). In analogy it seems to be promising to study moduli spaces of stable sheaves on higher-dimensional Hyperkähler manifolds. In this note we present examples of stable sheaves on the second Hilbert scheme of a K3 surface which is one of the two prototypes of four-dimensional compact Hyperkähler manifolds.

After introducing some notation in Section 1 we prove the theorem in Section 2. Finally we calculate the Chern classes of $\mathcal{L}^{[2]}$ in Section 3.

\section{Some notation.}

Let $\imath_{\Delta}: \Delta \hookrightarrow S \times S$ be the diagonal. Denote by $\sigma: \widetilde{S \times S} \rightarrow S \times S$ the blowup of $S \times S$ in $\Delta$. The natural action of the symmetric group $\widetilde{S}_{2}$ on $S \times S$ extends to a holomorphic action on $\widetilde{S \times S}$ and $\operatorname{Hilb}^{2}(S)=\widetilde{S \times S} / \widetilde{S}_{2}$. Let $l_{D}: D \hookrightarrow \widetilde{S \times S}$ be the exceptional divisor of $\sigma$.

In the following diagram we summarize the situation and, at the same time, give names to the various natural maps.

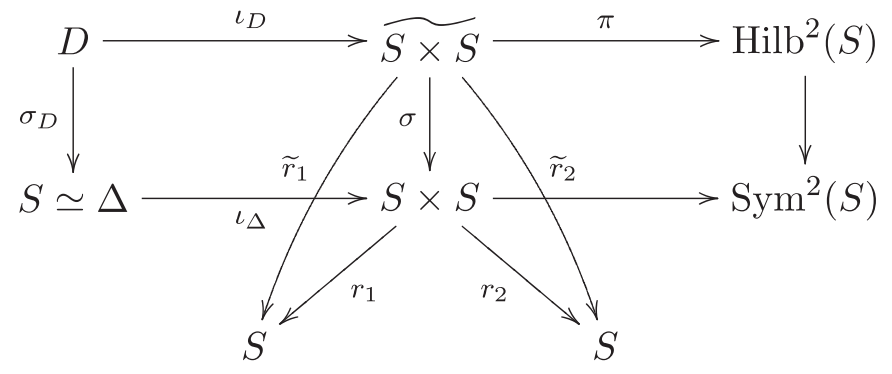

Given an ample hypersurface $H \subset S$, for $N$ sufficiently large the divisor $\widetilde{H}_{N}:=N\left(\widetilde{r}_{1}^{*} H+\widetilde{r}_{2}^{*} H\right)-D$ is ample on $S \times S$. Moreover, this divisor is invariant under the action of $\Xi_{2}$ on the divisor class group of $S \times S$, hence it is of the form $\pi^{*} H_{N}$ for an ample divisor $H_{N}$ on $\operatorname{Hilb}^{2}(S)$. 
Finally we recall the notion of $\mu$-stability. Let $Y$ be a smooth, projective variety, polarized by an ample divisor $H$. Let $\mathcal{E}$ be a torsion-free coherent $\mathcal{O}_{Y}$-module. Then the slope of $\mathcal{E}$ with respect to $H$ is defined as

$$
\mu_{H}(\mathcal{E}):=\frac{\int_{Y} c_{1}(\mathcal{E})[H]^{\operatorname{dim}(Y)-1}}{\operatorname{rk}(\mathcal{E})} .
$$

The sheaf $\mathcal{E}$ is called $\mu_{H}$-stable if for any subsheaf $\mathcal{F} \subset \mathcal{E}$ with $0<\operatorname{rk}(\mathcal{F})<\operatorname{rk}(\mathcal{E})$ we have $\mu_{H}(\mathcal{F})<\mu_{H}(\mathcal{E})$.

\section{Proof of the Theorem.}

If $\mathcal{L}^{[2]}$ had a destabilizing subsheaf, then by passing to the reflexive hull we see that there would exist a destabilizing line bundle. Thus, any destabilizing subsheaf of $\mathcal{L}^{[2]}$ on $\operatorname{Hilb}^{2}(S)$ with respect to $H_{N}$ induces a destabilizing sub-line bundle of $\mathcal{E}:=\pi^{*} \mathcal{L}^{[2]}$ on $\widetilde{S \times S}$ with respect to $\widetilde{H}_{N}$. We will show that $\mathcal{E}$ is $\mu_{\tilde{H}_{N}}$-stable. This will finish the proof of the theorem.

For $i=1,2$, put $\mathcal{L}_{i}:=\widetilde{r}_{i}^{*} \mathcal{L}$ and let $\mathcal{L}_{D}:=l_{D, *} \sigma_{D}^{*} \mathcal{L}$. Consider the fundamental short exact sequence (see e.g. the proof of [D, Prop. 2.3])

$$
0 \rightarrow \mathcal{E} \rightarrow \mathcal{L}_{1} \oplus \mathcal{L}_{2} \rightarrow \mathcal{L}_{D} \rightarrow 0
$$

where the surjection on the right is given by $\left(s_{1}, s_{2}\right) \mapsto s_{1 \mid D}-s_{2 \mid D}$. This sequence shows that $\mathrm{c}_{1}(\mathcal{E})=\mathrm{c}_{1}\left(\mathcal{L}_{1}\right)+\mathrm{c}_{1}\left(\mathcal{L}_{2}\right)-[D]$. Moreover, we deduce that $\mathcal{E}$ contains the line bundles $\mathcal{L}_{1}(-D)$ and $\mathcal{L}_{2}(-D)$.

Let now $\mathcal{A} \subset \mathcal{E}$ be an arbitrary sub-line bundle. Then $\mathcal{A}$ has one of the following three properties:

1.) $\mathcal{A} \subset \mathcal{L}_{1}(-D)$,

2.) $\mathcal{A} \subset \mathcal{L}_{2}(-D)$,

3.) $\mathcal{A} \not \subset \mathcal{L}_{1}(-D)$ and $\mathcal{A} \not \subset \mathcal{L}_{2}(-D)$.

We will prove that there exist $N_{1}, N_{2}, N_{3} \in \mathbb{N}$ such that for all $\mathcal{A} \subset \mathcal{E}$ with property $i=1,2$ or 3 and for all $N \geq N_{i}$ we have

$$
\mu_{\tilde{H}_{N}}(\mathcal{A})<\mu_{\tilde{H}_{N}}(\mathcal{E}) .
$$

Assume first that we are in case 1.), i.e. that $\mathcal{A} \subset \mathcal{L}_{1}(-D)$. Choose a natural number $N_{1} \geq 4$ (this will be useful in (11) below) such that $\widetilde{H}_{N}$ is ample for all $N \geq N_{1}$. Then $\mu_{\widetilde{H}_{N}}(\mathcal{A}) \leq \mu_{\widetilde{H}_{N}}\left(\mathcal{L}_{1}(-D)\right.$ ) for all $N \geq N_{1}$. Let $\alpha_{i}:=\mathrm{c}_{1}\left(\mathcal{L}_{i}\right)$. Then $\mathrm{c}_{1}(\mathcal{E})=\alpha_{1}+\alpha_{2}-[D]$ and therefore 
$(4)$

$$
\begin{aligned}
& \mu_{\widetilde{H}_{N}}(\mathcal{E})-\mu_{\widetilde{H}_{N}}(\mathcal{A}) \geq \mu_{\widetilde{H}_{N}}(\mathcal{E})-\mu_{\tilde{H}_{N}}\left(\mathcal{L}_{1}(-D)\right) \\
& =\int_{S \times S}\left(\frac{\alpha_{1}+\alpha_{2}-[D]}{2}-\left(\alpha_{1}-[D]\right)\right)\left[\widetilde{H}_{N}\right]^{3} \\
& =\int_{S \times S} \frac{\alpha_{2}-\alpha_{1}}{2}\left[\widetilde{H}_{N}\right]^{3}+\underset{S \times S}{\int} \frac{[D]}{2}\left[\widetilde{H}_{N}\right]^{3} \\
& =0+\underset{S \times S}{\int} \frac{[D]}{2}\left[\widetilde{H}_{N}\right]^{3} \\
& >0
\end{aligned}
$$

for all $N \geq N_{1}$ because $D$ is effective and $\widetilde{H}_{N}$ is ample.

An analogous reasoning applies in case 2.) with $N_{2}=N_{1}$.

In case 3.) we proceed in two steps. First we show that

$$
\mu_{F}(\mathcal{A})<\mu_{F}(\mathcal{E})
$$

where $F=\widetilde{r}_{1}^{*} H+\widetilde{r}_{2}^{*} H$ and $\mu_{F}$ is the slope with respect to the nef divisor $F$, defined as in (1). Then we use an asymptotic argument to complete the proof.

To prove (5), we will consider two divisors in $|M F|$ for an appropriate $M>0$ which intersect along a reducible surface. Then we reduce our computation to the irreducible components of these surfaces.

Choose $M$ sufficiently positive such that the linear system $|M H|$ contains two distinct, smooth curves $C$ and $C^{\prime}$ which intersect transversely and which satisfy

$$
\operatorname{deg}\left(\mathcal{L}_{\mid C}\right)=\operatorname{deg}\left(\mathcal{L}_{\mid C^{\prime}}\right)=\int_{S} \mathrm{c}_{1}(\mathcal{L})[M H] \geq 2 .
$$

Denote by

$$
G_{1}:=(C \times S) \cup\left(S \times C^{\prime}\right) \text { and by } G_{2}:=\left(C^{\prime} \times S\right) \cup(S \times C),
$$

let $\widetilde{G}_{1}$ and $\widetilde{G}_{2}$ be their strict transforms under $\sigma: \widetilde{S \times S} \rightarrow S \times S$. Note that $\widetilde{G}_{i} \in|M F|$ because each component of $G_{i}$ meets the center of the blowup, namely the diagonal of $S \times S$, along a curve. The intersection $\widetilde{G}_{1} \cap \widetilde{G}_{2}$ is the 
disjoint union of the four smooth surfaces

$$
\begin{gathered}
T_{1}:=\left(C \cap \widetilde{C^{\prime}}\right) \times S, \quad T_{2}:=S \times \widetilde{\left(\widetilde{C \cap} C^{\prime}\right),} \\
T_{3}:=\widetilde{C \times C}, \quad T_{4}:=C^{\prime} \times C^{\prime},
\end{gathered}
$$

where for any subvariety $Y \subsetneq S \times S$ we write $\widetilde{Y}$ for the strict transform of $Y$ under $\sigma: S \times S \rightarrow S \times S$. Then for the $M F$-slope of any coherent sheaf $\mathcal{F}$ we find

$$
\mu_{M F}(\mathcal{F}):=\frac{\int{ }_{S \times S} c_{1}(\mathcal{F})[M F]^{3}}{\operatorname{rk}(\mathcal{F})}=\sum_{i=1}^{4} \frac{\operatorname{deg}_{T_{i}}(\mathcal{F})}{\operatorname{rk}(\mathcal{F})}
$$

where $\operatorname{deg}_{T_{i}}(\mathcal{F}):=\int_{T_{i}} \mathrm{c}_{1}\left(\mathcal{F}_{\mid T_{i}}\right)[M F]_{\mid T_{i}}$. We will show that

$$
\operatorname{deg}_{T_{i}}(\mathcal{A}) \leq \frac{\operatorname{deg}_{T_{i}}(\mathcal{E})}{2}
$$

for $i=1, \ldots, 4$ with strict inequality for $i=3,4$. This will conclude the proof of (5).

$i=1$ : The surface $T_{1}$ is a disjoint union of surfaces of the form $S_{p}:=\{p\} \times S, p \in S$ running over the finite set $C \cap C^{\prime}$. Since the fundamental class of $S_{p}$ does not vary for different $p \in S$, we fix an arbitrary point $p_{0} \in S$ and we get for any coherent sheaf $\mathcal{F}$ on $S \times S$

$$
\operatorname{deg}_{T_{1}}(\mathcal{F})=\sharp\left(C \cap C^{\prime}\right) \operatorname{deg}_{S_{p_{0}}}(\mathcal{F}) .
$$

Note that $S_{p_{0}}$ is isomorphic to the blow-up of $S$ in $p_{0}$. Denote by $\sigma_{p_{0}}: S_{p_{0}} \rightarrow S$ the blow-down and by $E_{p_{0}} \subset S_{p_{0}}$ the exceptional divisor. Then

$$
\mathrm{c}_{1}\left(\mathcal{E}_{\mid S_{p_{0}}}\right)=\sigma_{p_{0}}^{*} \mathrm{c}_{1}(\mathcal{L})-\left[E_{p_{0}}\right]
$$

because $\mathrm{c}_{1}(\mathcal{E})=\widetilde{r}_{1}^{*} \mathrm{c}_{1}(\mathcal{L})+\widetilde{r}_{2}^{*} \mathrm{c}_{1}(\mathcal{L})-[D]$ and because $\widetilde{r}_{1}^{*} \mathcal{L}_{\mid S_{p_{0}}}=\mathcal{O}_{S_{p_{0}}}$. Now suppose that

$$
2 \operatorname{deg}_{T_{1}}(\mathcal{A})>\operatorname{deg}_{T_{1}}\left(\pi^{*} \mathcal{L}^{[2]}\right)
$$

Then we would get

$$
2 \int_{S_{p_{0}}} \mathrm{c}_{1}\left(\mathcal{A}_{\mid S_{p_{0}}}\right)[M F]_{\mid S_{p_{0}}}>\int_{S_{p_{0}}}\left(\sigma_{p_{0}}^{*} c_{1}(\mathcal{L})-\left[E_{p_{0}}\right]\right)[M F]_{\mid S_{p_{0}}} \geq 0
$$

because $[M F]_{\mid S_{p_{0}}}$ is a nef class on $S_{p_{0}}$ and because $\sigma_{p_{0}}^{*} \mathcal{L} \otimes \mathcal{O}\left(-E_{p_{0}}\right)$ is the line bundle of an effective divisor on $S_{p_{0}}$. Indeed, since $h^{0}(\mathcal{L}) \geq 2$, there exists a divisor $K \in|\mathcal{L}|$ with $p_{0} \in \operatorname{supp}(K)$. Then the strict transform $\widetilde{K}$ of 
$\underset{\widetilde{K}}{K}$ is in the linear system $\left|\sigma_{p_{0}}^{*} \mathcal{L} \otimes \mathcal{O}\left(-k E_{p_{0}}\right)\right|$ for some $k \geq 1$. Thus, $\widetilde{K}+(k-1) E_{p_{0}}$ is an effective divisor with line bundle $\sigma_{p_{0}}^{*} \mathcal{L} \otimes \mathcal{O}\left(-E_{p_{0}}\right)$.

By (2), $\mathcal{A}_{\mid S_{p_{0}}} \subset\left(\mathcal{L}_{1} \oplus \mathcal{L}_{2}\right)_{\mid S_{p_{0}}}=\mathcal{O}_{S_{p_{0}}} \oplus \sigma_{p_{0}}^{*} \mathcal{L}$. Now, since $\mathcal{A}_{\mid S_{p_{0}}}$ has positive $F_{\mid S_{p_{0}}}$-slope, the composition $\mathcal{A}_{\mid S_{p_{0}}} \rightarrow \mathcal{O}_{S_{p_{0}}} \oplus \sigma_{p_{0}}^{*} \mathcal{L} \rightarrow \mathcal{O}_{S_{p_{0}}}$ must be zero. Since we chose $p_{0} \in S$ randomly, assumption (7) implies that this composition is zero for all $p \in S$. On the other hand, all $x \in S \times S$ lie on some $S_{p}$. This shows that the composition

$$
\mathcal{A} \rightarrow \mathcal{L}_{1} \oplus \mathcal{L}_{2} \rightarrow \mathcal{L}_{1}
$$

is zero. But then the short exact sequence (2) implies that $\mathcal{A} \subset \widetilde{r}_{2}^{*} \mathcal{L}(-D)$, because the surjection $\mathcal{L}_{1} \oplus \mathcal{L}_{2} \rightarrow \mathcal{L}_{D}$ is given by $\left(s_{1}, s_{2}\right) \mapsto s_{1 \mid D}-s_{2 \mid D}$. This is a contradiction to the assumption that $\mathcal{A}$ satisfies 3.).

$i=2$ : analogous to $i=1$.

$i=3$ : Note that $C \times C$ is isomorphic to $C \times C$ and that

$$
M F \underset{\mid C \times C}{\underset{1}{C}}=M\left(p_{1}^{*} H_{\mid C}+p_{2}^{*} H_{\mid C}\right)
$$

where $p_{i}: C \times C \rightarrow C$ are the projections. Moreover, it is easily checked that

$$
\left(\pi^{*} \mathcal{L}^{[2]}\right) \underset{\mid C \times C}{\widetilde{C}}=\pi_{C}^{*} \mathcal{L}_{\mid C}^{[2]}
$$

where $\pi_{C}: C \times C \rightarrow \operatorname{Hilb}^{2}(C)$ is the natural projection and $\mathcal{L}_{\mid C}^{[2]}$ is the tautological vector bundle associated with $\mathcal{L}_{\mid C}$ on $\operatorname{Hilb}^{2}(C)$. Using (6), we can apply [Mi], Cor. 4.3.3 which says that $\pi_{C}^{*} \mathcal{L}_{[C}^{[2]}$ is a stable vector bundle on $C \times C$. This concludes the case $i=3$.

$i=4$ : analogous to $i=3$.

Thus, we have proved (5). To conclude the proof of (3) we define for $n \in \mathbb{N}$ the linear function

$$
\varphi_{n}: K^{0}(\operatorname{Coh}(\widetilde{S \times S})) \rightarrow Q, \quad \mathcal{F} \mapsto \sum_{i=0}^{2} n^{i} \frac{3 !}{i !(3-i) !} \underset{S \times S}{\int} c_{1}(\mathcal{F})[F]^{i}\left[\widetilde{H}_{N_{1}}\right]^{3-i} .
$$

Then noting that $\widetilde{H}_{n+N_{1}}=n F+\widetilde{H}_{N_{1}}$, we get for all $\mathcal{F} \in \operatorname{Coh}(\widetilde{S \times S})$

$$
\mu_{\widetilde{H}_{n+N_{1}}}(\mathcal{F})=n^{3} \mu_{F}(\mathcal{F})+\frac{\varphi_{n}(\mathcal{F})}{\operatorname{rk}(\mathcal{F})} .
$$

Inequality (5) implies that there exists a positive constant $k \in \mathbb{R}_{>0}$ such that for all sub-line bundles $\mathcal{A} \subset \mathcal{E}$ with property 3.) we have

$$
\mu_{F}(\mathcal{E})-\mu_{F}(\mathcal{A}) \geq k .
$$

This is because $\mu_{F}$ takes integer values on line bundles. 
We will now show that $\varphi_{n}(\mathcal{A})<\varphi_{n}(\mathcal{E})$. To see this, we first prove that $\mathcal{A} \cap \mathcal{L}_{1}(-D)=0$. Indeed, otherwise the torsion-free sheaf $\mathcal{A}+\mathcal{L}_{1}(-D)$ would be of rank 1 as follows from the short exact sequence

$$
0 \rightarrow \mathcal{A} \cap \mathcal{L}_{1}(-D) \rightarrow \mathcal{A} \oplus \mathcal{L}_{1}(-D) \rightarrow \mathcal{A}+\mathcal{L}_{1}(-D) \rightarrow 0 .
$$

Then the reflexive hull $\mathcal{A}^{\prime}$ of $\mathcal{A}+\mathcal{L}_{1}(-D)$ would be a sub-line bundle of $\mathcal{E}$ which again would have property 3.) because $\mathcal{A} \subset \mathcal{A}^{\prime}$. From (5) we deduce $\mu_{F}\left(\mathcal{A}^{\prime}\right)<\mu_{F}(\mathcal{E})$. On the other hand

$$
\begin{aligned}
\mu_{F}\left(\mathcal{L}_{1}(-D)\right) & =\int_{S \times S}\left(\alpha_{1}-[D]\right)[F]^{3} \\
& =\int_{S \times S} \alpha_{1}[F]^{3} \\
& =\int_{S \times S} \frac{\alpha_{1}+\alpha_{2}}{2}[F]^{3} \\
& =\int_{S \times S} \frac{\alpha_{1}+\alpha_{2}-[D]}{2}[F]^{3} \\
& =\mu_{F}(\mathcal{E}) .
\end{aligned}
$$

Here, we used that the integral over a cohomology class of degree 6 on $D$ which is pulled back from $\triangle$ vanishes. Using that $\mathcal{L}_{1}(-D) \subset \mathcal{A}^{\prime}$ and that $F$ is nef, we get a chain of inequalities

$$
\mu_{F}(\mathcal{E})=\mu_{F}\left(\mathcal{L}_{1}(-D)\right) \leq \mu_{F}\left(\mathcal{A}^{\prime}\right)<\mu_{F}(\mathcal{E}) .
$$

This is a contradiction, whence $\mathcal{A} \cap \mathcal{L}_{1}(-D)=0$.

Then we have a short exact sequence

$$
0 \rightarrow \mathcal{A} \oplus \mathcal{L}_{1}(-D) \rightarrow \mathcal{E} \rightarrow \mathcal{Q} \rightarrow 0
$$

where $\mathcal{Q}$ is a torsion sheaf. It follows that $\mathrm{c}_{1}(\mathcal{Q})$ is either zero or effective. Since $\varphi_{n}$ involves only products of the nef divisor $F$ and the ample divisor $\widetilde{H}_{N_{1}}$, this implies that $\varphi_{n}(\mathcal{Q}) \geq 0$.

We claim that there exists $n_{1} \in \mathbb{N}$ such that for $n \geq n_{1}$ we have $\varphi_{n}\left(\mathcal{L}_{1}(-D)\right)>0$. To see this, it is enough to show that the $n^{2}$-term of 
$\varphi_{n}\left(\mathcal{L}_{1}(-D)\right)$ is positive. We have

$$
\begin{aligned}
\mathrm{c}_{1}\left(\mathcal{L}_{1}(-D)\right)[F]^{2}\left[\widetilde{H}_{N_{1}}\right] & =\left(\alpha_{1}-[D]\right)[F]^{2}\left(N_{1}[F]-[D]\right) \\
& =N_{1} \alpha_{1}[F]^{3}+[F]^{2}[D]^{2}-[D]\left(N_{1}[F]^{3}+\alpha_{1}[F]^{2}\right) .
\end{aligned}
$$

If $q$ denotes the intersection product on $S$, then we obtain

$$
\begin{aligned}
\int_{S \times S} & N_{1} \alpha_{1}[F]^{3}+[F]^{2}[D]^{2}-[D]\left(N_{1}[F]^{3}+\alpha_{1}[F]^{2}\right) \\
& =N_{1} \int_{S \times S} r_{1}^{*} c_{1}(\mathcal{L})\left(r_{1}^{*}[H]+r_{2}^{*}[H]\right)^{3} \\
& +\int_{D}[F]^{2} \xi-\sigma_{D}^{*} \underbrace{\left\{\left(r_{1}^{*}[H]+r_{2}^{*}[H]\right)^{2}\left(N_{1}\left(r_{1}^{*}[H]+r_{2}^{*}[H]\right)+r_{1}^{*} c_{1}(\mathcal{L})\right)\right\}_{\mid A}}_{=0} \\
& =3 N_{1} \int_{S \times S} r_{1}^{*} c_{1}(\mathcal{L}) r_{1}^{*}[H] r_{2}^{*}[H]^{2}-2 \int_{S}(2[H])^{2} \\
& =\left(3 N_{1} q\left(c_{1}(\mathcal{L}),[H]\right)-4\right) q([H],[H]) \\
& >0 .
\end{aligned}
$$

Here, $r_{i}: S \times S \rightarrow S$ are the projections and $\xi=[D]_{\mid D}=\mathrm{c}_{1}\left(\mathcal{O}_{\mathrm{P}\left(\mathcal{N}_{A \mid S \times S}\right)}(-1)\right)$. We use that $\int_{D}\left(\sigma_{D}^{*} \alpha\right) \xi=-\int_{S} \alpha$ for $\alpha \in H^{*}(S, \mathrm{Q})$, that $N_{1} \geq 4$ and that $\mathrm{c}_{1}(\mathcal{L})$ is an effective class on $S$. This proves the existence of $n_{1}$ with $\varphi_{n}\left(\mathcal{L}_{1}(-D)\right)>0$ for all $n \geq n_{1}$.

Now by (10) we have

$$
\varphi_{n}(\mathcal{A})=\varphi_{n}(\mathcal{E})-\varphi_{n}(\mathcal{Q})-\varphi_{n}\left(\mathcal{L}_{1}(-D)\right)<\varphi_{n}(\mathcal{E})
$$

for all $n \geq n_{1}$.

Putting together (8), (9) and (12) we find for $n \geq n_{1}$ and for all line bundles $\mathcal{A} \subset \mathcal{E}$ with property 3.)

$$
\begin{aligned}
\mu_{\widetilde{H}_{n+N_{1}}}(\mathcal{E})-\mu_{\widetilde{H}_{n+N_{1}}}(\mathcal{A}) & =n^{3}\left(\mu_{F}(\mathcal{E})-\mu_{F}(\mathcal{A})\right)+\frac{\varphi_{n}(\mathcal{E})}{2}-\varphi_{n}(\mathcal{A}) \\
& >n^{3} k-\frac{\varphi_{n}(\mathcal{E})}{2} .
\end{aligned}
$$

Now since $k>0$ and since $\varphi_{n}(\mathcal{E})$ is a polynomial of degree 2 in $n$, there exists $n_{2} \geq n_{1}$ such that $n^{3} k-\frac{\varphi_{n}(\mathcal{E})}{2}>0$ for all $n \geq n_{2}$. Therefore, with $N_{3}:=$ $N_{1}+n_{2}$, inequality (3) is satisfied for $i=3$. This completes the proof. 


\section{The Chern character.}

In this section we express the Chern classes of $\mathcal{L}^{[2]}$ in terms of $\mathrm{c}_{2}\left(\operatorname{Hilb}^{2}(S)\right)$, of the symmetric product of $\mathrm{c}_{1}(\mathcal{L})$ and of the fundamental class of the exceptional divisor of the Hilbert-Chow morphism

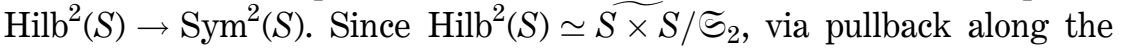
quotient morphism $\pi$ we get an identification $H^{*}\left(\operatorname{Hilb}^{2}(S), Q\right) \simeq$

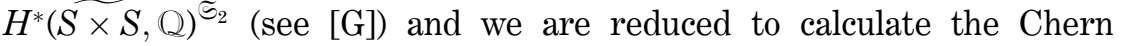
classes of $\pi^{*} \mathcal{L}^{[2]}$. By the short exact sequence (2) we get

$$
\begin{aligned}
& \pi^{*} \mathrm{c}_{1}\left(\mathcal{L}^{[2]}\right)=\alpha_{1}+\alpha_{2}-[D] \text { and } \\
& \pi^{*} \mathrm{c}_{2}\left(\mathcal{L}^{[2]}\right)=\mathrm{c}_{2}\left(\mathcal{L}_{1} \oplus \mathcal{L}_{2}\right)-\mathrm{c}_{2}\left(\mathcal{L}_{D}\right)-\mathrm{c}_{1}\left(\mathcal{L}^{[2]}\right) \mathrm{c}_{1}\left(\mathcal{L}_{D}\right)=\alpha_{1} \alpha_{2}-\frac{1}{2}\left(\alpha_{1}+\alpha_{2}\right)[D],
\end{aligned}
$$

where as above $\alpha_{i}=\widetilde{r}_{i}^{*} \mathrm{c}_{1}(\mathcal{L})$ and where we used the Grothendieck-Riemann-Roch theorem to calculate $c_{2}\left(\mathcal{L}_{D}\right)$.

Using the tangent bundle sequence for the ramified covering $\pi: \widetilde{S \times S \rightarrow \operatorname{Hilb}^{2}(S)}$

$$
0 \rightarrow \mathcal{T} \underset{S \times S}{\sim} \rightarrow \pi^{*} \mathcal{T}_{\mathrm{Hilb}^{2}(S)} \rightarrow \mathcal{O}_{D}(2 D) \rightarrow 0
$$

we get

$$
\pi^{*} \mathrm{c}_{1}\left(\operatorname{Hilb}^{2}(S)\right)=\mathrm{c}_{1}(\widetilde{S \times S})+\mathrm{c}_{1}\left(\mathcal{O}_{D}(2 D)\right)
$$

$$
\pi^{*} \mathrm{c}_{2}\left(\operatorname{Hilb}^{2}(S)\right)=\mathrm{c}_{2}(\widetilde{S \times S})+\mathrm{c}_{2}\left(\mathcal{O}_{D}(2 D)\right)+\mathrm{c}_{1}(\widetilde{S \times S}) \mathrm{c}_{1}\left(\mathcal{O}_{D}(2 D)\right) .
$$

Now the formulae for Chern classes of blow-ups (cf. [Fu, Ex. 15.4.3]) yield

$$
\begin{aligned}
\mathrm{c}_{1}(\widetilde{S \times S}) & =\sigma^{*} \mathrm{c}_{1}(S \times S)-[D] \\
\mathrm{c}_{2}(\widetilde{S \times S}) & =\sigma^{*}\left(\mathrm{c}_{2}(S \times S)\right)-\left(l_{D}\right)_{*} \sigma_{D}^{*} \mathrm{c}_{1}(\Delta)-[D]^{2} \\
& =\sigma^{*} \mathrm{c}_{2}(S \times S)-\frac{1}{2} \sigma^{*} \mathrm{c}_{1}(S \times S)[D]-[D]^{2} .
\end{aligned}
$$

By the Grothendieck-Riemann-Roch formula

$$
\begin{aligned}
& \mathrm{c}_{1}\left(\mathcal{O}_{D}(2 D)\right)=[D] \\
& \mathrm{c}_{2}\left(\mathcal{O}_{D}(2 D)\right)=-[D]^{2} .
\end{aligned}
$$

Combining (13), (14) and (15) we obtain

$$
\pi^{*} c_{1}\left(\operatorname{Hilb}^{2}(S)\right)=\sigma^{*} c_{1}(S \times S)
$$


and

$$
\begin{aligned}
\pi^{*} \mathrm{c}_{2}\left(\operatorname{Hilb}^{2}(S)\right)= & \sigma^{*} \mathrm{c}_{2}(S \times S)-\frac{1}{2} \sigma^{*} \mathrm{c}_{1}(S \times S)[D]-[D]^{2}-[D]^{2} \\
& +\sigma^{*} \mathrm{c}_{1}(S \times S)[D]-[D]^{2} \\
= & \sigma^{*} \mathrm{c}_{2}(S \times S)+\frac{1}{2} \sigma^{*} \mathrm{c}_{1}(S \times S)[D]-3[D]^{2} \\
= & -\sigma^{*} \operatorname{ch}_{2}(S \times S)+\frac{1}{2} \sigma^{*} c_{1}^{2}(S \times S) \\
& +\frac{1}{2} \pi^{*} \mathrm{c}_{1}\left(\operatorname{Hilb}^{2}(S)\right)[D]-3[D]^{2} \\
= & -\int_{S} \operatorname{ch}_{2}(S) \cdot\left(\sigma^{*}[\Delta]^{4,0}+\sigma^{*}[\Delta]^{0,4}\right) \\
& +\pi^{*}\left(\frac{\mathrm{c}_{1}\left(\operatorname{Hilb}^{2}(S)\right) \delta+\mathrm{c}_{1}^{2}\left(\mathrm{Hilb}^{2}(S)\right)}{2}-3 \delta^{2}\right) .
\end{aligned}
$$

Here, $[\Delta]^{i, j}$ refers to the $(i, j)$-th Künneth factor of fundamental class of the diagonal $\Delta \subset S \times S$ and $\delta \in H^{2}\left(\operatorname{Hilb}^{2}(S), Q\right)$ is the class with $\pi^{*} \delta=[D]$. If $\int_{S} \operatorname{ch}_{2}(S) \neq 0$, this implies that

$$
\begin{aligned}
\alpha_{1} \alpha_{2}= & \frac{1}{2}\left(\left(\alpha_{1}+\alpha_{2}\right)^{2}-\alpha_{1}^{2}-\alpha_{2}^{2}\right) \\
= & \frac{1}{2}\left(\left(\alpha_{1}+\alpha_{2}\right)^{2}-q\left(c_{1}(\mathcal{L}), c_{1}(\mathcal{L})\right) \cdot \sigma^{*}\left([\Delta]^{4,0}+[\Delta]^{0,4}\right)\right) \\
= & \frac{\left(\alpha_{1}+\alpha_{2}\right)^{2}}{2}+\frac{q\left(c_{1}(\mathcal{L}), c_{1}(\mathcal{L})\right)}{2 \int_{S} \operatorname{ch}(S)} \\
& \left\{c_{2}\left(\operatorname{Hilb}_{2}^{2}(S)\right)-\frac{\mathrm{c}_{1}^{2}\left(\operatorname{Hilb}^{2}(S)\right)+\mathrm{c}_{1}\left(\operatorname{Hilb}^{2}(S)\right) \delta}{2}+3 \delta^{2}\right\} .
\end{aligned}
$$

As above, $q$ denotes the intersection product on $H^{2}(S, \mathrm{Q})$.

Let $\varphi: H^{2}(S, Q) \rightarrow H^{2}\left(\operatorname{Hilb}^{2}(S), Q\right)$ be the homomorphism which is determined by

$$
\pi^{*} \varphi(\beta)=\widetilde{r}_{1}^{*} \beta+\widetilde{r}_{2}^{*} \beta \text { for all } \beta \in H^{2}(S, \mathrm{Q}) .
$$


Summarizing the above discussion we have achieved an expression of the Chern classes of $\mathcal{L}^{[2]}$ in terms of $\mathrm{c}_{1}\left(\operatorname{Hilb}^{2}(S)\right)$ and $\mathrm{c}_{2}\left(\operatorname{Hilb}^{2}(S)\right)$, of $\varphi\left(\mathrm{c}_{1}(\mathcal{L})\right)$ and of $\delta$ :

Proposition. i) Assume that $\int_{S} \operatorname{ch}_{2}(S) \neq 0$. Then the Chern classes of $\mathcal{L}^{[2]}$ are

$$
\begin{aligned}
\mathrm{c}_{1}\left(\mathcal{L}^{[2]}\right) & =\varphi\left(\mathrm{c}_{1}(\mathcal{L})\right)-\delta \text { and } \\
\mathrm{c}_{2}\left(\mathcal{L}^{[2]}\right) & =\frac{\varphi\left(\mathrm{c}_{1}(\mathcal{L})\right)^{2}-\varphi\left(\mathrm{c}_{1}(\mathcal{L})\right) \delta}{2} \\
& +\frac{q\left(\mathrm{c}_{1}(\mathcal{L}), \mathrm{c}_{1}(\mathcal{L})\right)}{2 \int_{S} \operatorname{ch}_{2}(S)}\left\{\mathrm{c}_{2}\left(\operatorname{Hilb}^{2}(S)\right)-\frac{\mathrm{c}_{1}^{2}\left(\operatorname{Hilb}^{2}(S)\right)+\mathrm{c}_{1}\left(\operatorname{Hilb}^{2}(S)\right) \delta}{2}+3 \delta^{2}\right\} .
\end{aligned}
$$

ii) For $N \gg 0$, the bundle $\mathcal{L}^{[2]}$ satisfies the strict Bogomolov-Lübke inequality, that is

$$
\Delta\left(\mathcal{L}^{[2]}\right) H_{N}^{2}=\left(4 \mathrm{c}_{2}\left(\mathcal{L}^{[2]}\right)-\mathrm{c}_{1}^{2}\left(\mathcal{L}^{[2]}\right)\right) H_{N}^{2}>0 .
$$

In particular, $\mathcal{L}^{[2]}$ is not projectively flat.

Proof. It remains to show ii). Using the above calculations we find

$$
\pi^{*} \Delta\left(\mathcal{L}^{[2]}\right)=2 \alpha_{1} \alpha_{2}-\alpha_{1}^{2}-\alpha_{2}^{2}-[D]^{2}=\Delta\left(\mathcal{L}_{1} \oplus \mathcal{L}_{2}\right)-[D]^{2} .
$$

Now, $\mathcal{L}_{1} \oplus \mathcal{L}_{2}$ is $\mu_{\widetilde{H}_{N}}$-polystable, thus

$$
\Delta\left(\mathcal{L}_{1} \oplus \mathcal{L}_{2}\right) \widetilde{H}_{N}^{2} \geq 0 .
$$

On the other hand,

$$
-[D]^{2} \widetilde{H}_{N}^{2}=-\int_{D} \widetilde{H}_{N}^{2} \xi=4 N^{2} q(H, H)+O(N)>0 \text { for } N \gg 0 .
$$

Thus

$$
\Delta\left(\mathcal{L}^{[2]}\right) H_{N}^{2}=\pi^{*} \Delta\left(\mathcal{L}^{[2]}\right) \widetilde{H}_{N}^{2}>0
$$

for $N \gg 0$.

Acknowledgments. This paper is a part of my $\mathrm{Ph}$. D. thesis prepared at the University of Bonn. It is a great pleasure to thank my advisor Daniel Huybrechts for his constant support. I am also grateful to Luca Scala for helpful discussions on Chern characters of tautological vector bundles and to Ernesto Mistretta for explaining to me his results about stable vector bundles on symmetric products of curves. 


\section{REFERENCES}

[D] G. Danila, Sur la cohomologie d'un fibré tautologique sur le schéma de Hilbert d'une surface, J. Alg. Geom., 10 (2001), pp. 247-280.

[Fo] J. Fogarty, Algebraic families on an algebraic surface, Am. J. Math., 90 (1968), pp. 511-521.

[Fu] W. Fulton, Intersection theory, Erg. Math., 3. Folge, Band 2, 2nd ed., Springer (1998).

[G] A. GRothendiEck, Sur quelques points d'algèbre homologique, Tôhoku Math. J., 9 (1957), pp. 119-221.

[GHJ] D. JoYce - D. HuYBrechts - M. Gross, Calabi-Yau manifolds and related geometries, Universitext, Springer (2002).

[HL] D. HuYBRECHTS - M. LEHN, The geometry of moduli spaces of sheaves, Cambridge Math. Libr., 2nd ed., Cambridge University Press (2010).

[Ma] E. Markman, On the monodromy of moduli spaces of sheaves on K3 surfaces, J. Alg. Geom., 17 (2008), pp. 29-99.

[Mi] E. MistRetTA, Some constructions around stability of vector bundles on projective varieties, $\mathrm{PhD}$ Thesis, Univ. Paris VII (2006).

[Mu] S. MukaI, On the moduli space of bundles on a K3 surface, In: Vector Bundles on Algebraic Varieties, Oxford University Press (1987), pp. 341413.

[OG] K. O'GRADY, The weight two Hodge structure of moduli spaces of sheaves on a K3 surface, J. Alg. Geom., 4 (1997), pp. 599-644.

Manoscritto pervenuto in redazione l'1 ottobre 2009. 